\title{
Guidelines for submissions
}

\section{Purpose}

The Colombian Applied Linguistics Journal, CALJ is published twice a year in June and December. It is mainly concerned with disseminating completed and ongoing research, pedagogical innovations, and theoretical reviews relevant to the field of applied linguistics and language education.

\section{Audience}

The Colombian Applied Linguistics Journal has a wide local and international audience. It is intended to inform the local, national and international community of educators interested in research on the teaching of English as a foreign language and on second/foreign language acquisition. It aims at informing teacher researchers in the areas of English as a Foreign Language (EFL), Spanish as a Foreign Language (SFL), Spanish as mother tongue, and applied linguistics, English teachers in primary, secondary, and institutions of higher education, including masters and Ph.D.'s and teachers and students of graduate and undergraduate teacher education programs in all public and private universities in Bogotá.

\section{National and International Exchanges}

Our publication circulates nationally at Universidad Tecnológica de Colombia in Tunja, Boyacá, at Universidad de Antioquia, Universidad del Valle, Universidad de Córdoba, Universidad Industrial de Santander, Universidad Nacional de Colombia in Bogotá, Universidad Tecnológia de Bolivar, Universidad del Norte, Universidad del Chocó, Universidad Surcolombiana and Universidad del Tolima, through academic exchanges with the institutions or through academic events that take place in different parts of the country. Private universities also receive our publication through annual memberships. CALJ is also received through international exchange agreements at Universidad Nacional (UNA) in Costa Rica, at USACH in Chile, at University of Lleida in Spain, and at University of Arizona, University of New Hampshire, Purdue University Calumet, University of Massachusetts-Amherst in the United States.

\section{Selection Process}

In order to guarantee a high quality process of selection and publication, the manuscripts received by the CALJ editor pass through a revision process prior to being approved. Once a manuscript is received the assistant to the editor does a first technical revision of the required sections for scientific articles and proceeds to classify it in one of the sections of the journal and the members of the editorial committee evaluate the contents of the article and assign two external evaluators. The articles that are not ready to be sent to evaluators are returned to the authors for revisions to the content or format of the article until if fulfils the requirements to proceed to assign evaluators. Once the article passes this first revision it is send to two external evaluators from the Editorial Review Board of the journal for a double blind peer review process. The evaluation can take from one to two months. In cases when there is a disagreement in the concepts provided by the two evaluators a third internal or external evaluator is required to provide an evaluative concept. The evaluators are invited to evaluate the manuscripts and they receive a copy of the article and a copy of the evaluation format. Once we receive the two concepts from the evaluators we contact the author and inform about the results of the evaluation. In the case the article requires minor revisions to its content as suggested by the evaluators the author(s) are given 2 weeks to do so. In the contrary, when the article is not accepted the assistant also informs the author immediately of the results of the evaluation. Regularly, from the moment a manuscript is received to the moment the authors are informed about the acceptance or rejection of a manuscript the process can take from 3 months.

\section{Specific Requirements}

- In order to be accepted for publication in CALJ, contributions should:

- Be original and interesting concerning the subject, methodology, and conclusions

- Be relevant to current research and theoretical implications

- Include knowledge of previous research in the same field

- Be scientifically rigorous and have great depth of analysis

- Be accurate in the use of concepts and terminology

- Have correct formatting, style and organization according to APA style

- Be well-written with sophistication and conciseness

- Be without bias or prejudice

\section{Creative Commons LICENSE}

The Colombian Applied Linguistics Journal is licensed under a Creative Commons Attribution-Non Commercial-No Derivs 2.5 Colombia. Readers can read, copy and distribute the contents of this publication under the terms of Creative Commons, Colombia. For further information refer to: http:// creativecommons.org/licenses/by-nc-nd/2.5/co/

\section{Types of Articles}

CALJ welcomes the following types of articles as required by Colciencias:

1. Research Articles: A document that details the original results of research projects. The structure of this type of article typically contains the following sections: title, abstract, key words, introduction, theoretical framework, methodology, analysis and discussion of results, conclusions, pedagogical implications, and references. Authors are encouraged to include graphic aides. Authors of research articles selected for publication will have to submit a separate form detailing the associated research project.

2. Reflections on Praxis: A document that shows research results on a specific topic from the author's analytical, interpretative, or critical perspective that presents an innovation with pedagogical implications. This type of article may include: theoretical considerations, pedagogical context, findings, discussion, context, etc. Authors are encouraged to include graphic aides. It should contain original sources.

3. Theoretical Discussion Papers: A document derived from research in which published or non-published research results in a scientific field are analyzed, systematized, and integrated in order to inform readers of the latest tendencies and developments. It typically presents a careful revision of literature with at least 50 references. This type of article may include: developments, factors, perspectives, context, literature review, debate, approaches, criticisms, etc. Authors are encouraged to include graphic aides. 
4. Short Articles: A brief document that preliminarily or partially presents original results of a scientific research study and generally requires prompt dissemination. This type of article may include: developments, factors, perspectives, context, implications, etc. Authors are encouraged to include graphic aides.

5. Case Reports: A document that presents the results of a study on a particular situation in order to disseminate the methodological and technical experiences used in a specific case. The report includes a systematic and commented literature review of similar cases. Authors are encouraged to include graphic aides.

6. Theme Reviews: A document resulting from a critical revision of literature related to a specific topic.

7. Book Review: Critical, analytical review, should provide some context for the work under consideration. This doesn't have to be extensive, not more that 2000 words, but should answer basic questions an informed reader. Always be specific. Provide evidence to back up your opinions.

\section{Form and Style}

Title page: Includes the title and the name, institutional affiliation, full postal address and e-mail address of each author in a separate word document from the article. Indicate the joint author to whom correspondence should be sent.

\begin{abstract}
An abstract written in English and Spanish (resumen) must be included. If the author is unable to translate an abstract into either language, please indicate this in your submission. The abstract should be between 150 and 200 words. Abstracts must clearly show the problem or issue under study, the theoretical perspective(s) under which the problem is examined, the methodology employed, and, finally, results and conclusions.
\end{abstract}

Key words: List four to six key words for the article. Write "key words:" in italics at the end of your abstract. Do not bold. Please include keywords (palabras claves) in Spanish as well as in English.

\section{Scientific articles regularly include four main sections:} Introduction (it includes the description of the problem and the theoretical support to the study), Methodology, Results, Discussion of Results and Conclusions.

Graphic Aids: Authors are highly encouraged to submit graphic aids with their articles. Graphic aids could include tables, diagrams, appendixes, illustrations, etc. that help the reader understand the article. They should include the corresponding reference citations. Please include graphic aides with high resolution (300dpi) in a separate word document. Where a graphic aide is to be inserted in the text, on a separate line write [INSERT Graphic Aid Name HERE]. Each graphic aid should be clearly labeled with number and caption. They should be numbered Figure 1, Figure 2, Table 1, Table 2, etc. Graphics may be modified or left out by the decision of the editor, according to the needs of the publication. Any changes will be discussed with the author before publication.
Length: Articles normally should be between 4,000 and 8,000 words (including the abstract, footnotes, references, tables, figures, appendixes and all other matter). The length of the manuscript should not exceed 8,000 words. Contributions which exceed these maximum lengths may be considered for publication in those cases in which their scientific relevance justifies it.

Software Requirements: All articles should be submitted in a Microsoft Word operating system, including Word 93-2007, Vista, or Windows 7.

Languages published: Papers are received in formal academic English or Spanish. Linguistic expressions from other cultural groups (i.e., Spanglish, slang, Cockney, AfroAmerican, Quechua, etc.) are welcome as samples and objects of research.

Bio-data and anonymity: All articles go through a peer-review process and are read anonymously by a member of CALJ's evaluation committee. Therefore, contributors are requested to include their full name and professional affiliation in a separate word document. In all cases, contributors are kindly asked to refrain from writing their names and professional affiliation in the body of their articles.

Guarantee of First Publication: Contributors will be asked to sign a letter acknowledging that the article sent to CALJ has been neither previously published in its submitted form or essentially similar version, nor sent elsewhere to be considered for print or electronic publication.

Publication Authorization Form: All contributors whose articles are accepted for publication will be required to sign an authorization form to agree that the Colombian Applied Linguistics Journal may publish the article on an Open Access database. Author(s) also agree that articles published in the Colombian Applied Linguistics Journal may not be reproduced, although they may request permission to reproduce published material. Signature of the form is a condition of publication and papers will not be passed to the publisher unless a signed form has been received.

Compensation: Authors of articles will receive two complimentary copies of CALJ in which their article appears. Permission and Consent: If the article contains extracts from other works, especially figures, tables, etc., please contact the authors and publishers (the holders of the copyright) before submitting the final version to seek permission to use their work. By submitting the article, authors warrant that they have obtained permission from the copyright holder to reproduce (in any printed or electronic format) material not written by the author and that the author has acknowledged the source. If primary data is to be included, research participants should have signed a consent form.

Submissions: The submission should be broken into three separate files: the title page, the article which has been blinded for review, and any graphic aides. Articles can be sent via email to caljournal.ud@gmail.com or maestria@ udistrital.edu.co. 
Waiver: Every article shall be subject to the review of the editorial committee. The editor reserves the right to make formal modifications to articles throughout the editing process.

\section{Contact:}

Communicate with the journal at caljournal.ud@gmail.com or by calling Tel: (571) 3238400 Ext. 6362

or through postal mail at:

CALJ, Editor Maestría en Lingüística Aplicada a la Enseñanza de Inglés

Universidad Distrital Francisco José de Caldas

Avenida Ciudad de Quito No. 64-81 Oficina 704

Bogotá, Colombia, Sur América

\section{Format}

Please follow the Publication Manual of the American Psychological Association (APA), Sixth Edition, 2010, for appropriate format. You might find the following links useful:

\section{APA Style Guide:}

http://www.apastyle.org/apa-style-help.aspx

APA Formatting and Style Guide:

http://owl.english.purdue.edu/owl/resource/560/1/

APA Paper sample:

http://owl.english.purdue.edu/owl/resource/560/18/

Font: Times New Roman, 12 points. Double space the entire document. Spaces between words or after full periods and colons should be a single space.

Margins: 1 " margins on every side

Title: Center, do not bold, use lower case, capitalize only the first letter of every word except prepositions, may use up to two lines.

Header: Must appear on every page of your paper. Write the title of your paper in capital letters in the top left margin. Number pages consecutively in the top right margin.

Headings: No more than two levels of heading below the title. Headings should not be numbered. The first level heading is bold, lowercase except for the first letter of every word. A second level heading is italics, also lowercase except for the first letter of every word.

Paragraphs: A line space should be left between paragraphs. The first line of new paragraphs should be indented, using a $1 / 4 \mathrm{~cm}$ indentation $\left(1 / 2^{\prime \prime}\right)$. Do not use a hard return at the end of a line in running text except at the end of a paragraph.

Page breaks: Do not insert page breaks in the text. Do not insert extra spacing to avoid widows or orphans. Page breaks will be different in the typeset proofs. Turn Hyphenation and Justification off.

Proofread and spell check your work when you have finished. Make a back-up of all your work. If you detect any virus on your machine, please inform all your CALJ contacts immediately.

\section{Documentation of Sources}

Please follow APA guidelines for appropriate documentation of sources in your paper and for your reference list. You might find the following links useful:

APA Reference List: http://owl.english.purdue.edu/owl/ resource/560/05/

APA In-Text Citations: http://owl.english.purdue.edu/owl/ resource/560/02/

The following are examples of how to list book, chapter in a book, article, government document, article in proceedings, article from an online periodical, etc, in the reference list.

\section{Book}

Calfee, R. C., \& Valencia, R. R. (1991). APA guide to

preparing manuscripts for journal publication. Washington, DC:

American Psychological Association.

\section{Chapter in book}

O'Neil, J. M., \& Egan, J. (1992). Men's and women's gender role journeys: A metaphor for healing, transition, and transformation. In B. R. Wainrib (Ed.), Gender issues across the life cycle (pp. 107-123). New York, NY: Springer.

\section{Article in Journal}

Harlow, H. F. (1983). Fundamentals for preparing psychology journal articles. Journal of Comparative and Physiological Psychology, 55, 893-896.

\section{Government document}

National Institute of Mental Health. (1990). Clinical training in serious mental illness (DHHS Publication No. ADM 90-1679). Washington, DC: U.S. Government Printing Office.

\section{Conference Proceedings}

Schnase, J. L., \& Cunnius, E. L. (Eds.). (1995). Proceedings from CSCL '95: The First International Conference on Computer Support for Collaborative Learning. Mahwah, NJ: Erlbaum.

\section{Article from an Online Periodical}

Author, A. A., \& Author, B. B. (Date of publication). Title of article. Title of Online Periodical, volume number(issue number if available). Retrieved from http://www. someaddress.com/full/url/

Footnotes: These should be kept to an absolute minimum and should be placed at the end of the main text. Do not use automatic footnote programs. Citations should appear within the text.

In-text references: These should appear in the body of the article, not in footnotes, giving the author's last name followed by the year and page number where relevant. Use double quotation marks for quoted material. Any quotation that runs for more than 40 words should be set off from the main paragraph and does not need quotation marks. Refer to APA for more information. 


\section{Guía para autores}

\section{Propósito}

La revista Colombian Applied Linguistics Journal, CALJ se publica dos veces al año en los meses de junio y diciembre. Su propósito principal es la difusión de investigaciones, terminadas y en curso, innovaciones pedagógicas, revisiones de temas teóricos relevantes al campo de estudios de la lingüística aplicada y la enseñanza de la lengua.

\section{Audiencia \\ La revista Colombian Applied Lingustics tiene una amplia audiencia regional, nacional e internacional. Su propósito es informar a la comunidad de educadores, nacionales e internacionales, Profesores de Inglés en primaria, secundaria e instituciones de educación superior incluyendo maestrías y doctorados (Ph.D.s) interesados en las investigaciones sobre la enseñanza del inglés como lengua extranjera (EFL), español como lengua extranjera (SFL), español como lengua materna, y lingüística aplicada sobre las publicaciones en estos temas. Nuestros lectores también son estudiantes y profesores de pregrado y posgrado de los programas de ciencias de la educación en universidades públicas y privadas de Bogotá.}

\section{Intercambios nacionales e internacionales}

Nuestra publicación circula nacionalmente en las siguientes universidades: Univesidad Tecnológica de Colombia en Tunja, Boyacá, Universidad de Antioquia, Universidad del Valle, Universidad de Córdoba, Universidad Industrial de Santander, Universidad Nacional de Colombia en Bogotá, Universidad Tecnológica de Bolívar, Universidad del Norte, Universidad del Chocó, Universidad Surcolombiana y la Universidad del Tolima; por medio de intercambios académicos con las instituciones o por medio de eventos académicos realizados en diferentes partes del país. Algunas universidades privadas reciben la publicación por medio de suscripciones anuales. La revista CALJ también tiene convenios de intercambio internacionales con la universidades: Universidad Nacional (UNA) en Costa Rica, USACH en Chile, la Universidad de Lleida en España, y las universidades de Arizona, New Hampshire, Purdue la Universidad Calumet, y la Universidad de MassachusettsAmherst en los Estados Unidos.

\section{Proceso de selección}

Para garantizar la calidad del proceso de selección y publicación, los manuscritos recibidos, se someten a una detallada revisión realizada por el comité editorial de la revista antes de ser aprobados. Una vez el manuscrito es recibido, el/la asistente del editor, hace una primera revisión técnica y formal de las secciones requeridas para artículos científicos, y procede a clasificarlo en una de las secciones de la revista. El comité editorial se reúne posteriormente a evaluar el contenido del artículo y asignarle dos evaluadores externos. Los artículos que no están listos para ser enviados a los evaluadores, son devueltos al autor para que realice las revisiones de contenido o forma del artículo hasta que cumpla con todos los requerimientos para asignarle los evaluadores.

Una vez el artículo pasa la primera etapa de revisión, es enviado a dos evaluadores externos miembros del comité evaluador de la revista para el proceso doblemente ciego de revisión por pares. El proceso de revisión puede llevar de uno a dos meses. En casos donde haya un desacuerdo en los conceptos proporcionados por los dos evaluadores un tercer evaluador interno o externo, será requerido para que proporcione un concepto de evaluación para el artículo. Los evaluadores son invitados a evaluar los manuscritos. Reciben una copia del artículo y un formato de evaluación. Una vez recibidos los dos conceptos de los evaluadores, se contacta al autor y se le informan los resultados de la evaluación. En el caso en el que el artículo requiera modificaciones menores en el contenido sugeridas por los evaluadores, los autores disponen de dos semanas para hacer los respectivos cambios. Por el contrario, cuando el artículo no es aceptado, el/la asistente de la revista notifica al autor de la decisión de los evaluadores. Regularmente, desde el momento en que se recibe el manuscrito al momento en que los autores son informados de la decisión de los evaluadores hay un lapso de tres meses.

\section{Contenido}

Colombian Applied Linguistics Journal, CALJ, se refiere principalmente a la difusión de las investigaciones terminadas y en curso, las innovaciones pedagógicas, teóricas y críticas pertinentes en el ámbito de la lingüística aplicada y enseñanza de lenguas.

Para la aceptación de una publicación en CALJ, las contribuciones deben:

- Ser originales e interesantes en relación con el tema, la metodología y las conclusiones.

- Ser relevantes en cuanto a la investigación en curso y las implicaciones teóricas.

- Incluir el conocimiento de investigaciones previas en el mismo campo.

- Ser científicamente riguroso y tener una gran profundidad de análisis.

- Ser preciso en el uso de conceptos y la terminología.

- Tener formato correcto, el estilo y organización de acuerdo con estilo de la Asociación Americana de Sicologia (APA).

- Estar bien escrito usando lenguaje académico especializado y conciso.

- Ser imparcial y evitar los prejuicios

\section{Licencia Creative commons}

Esta publicación tiene licencia de Creative Commons Reconocimiento-No comercial- Sin obras derivadas 2.5 Colombia. El lector podrá leer, copiar y distribuir los contenidos de esta publicación bajo los términos legales de Creative Commons, Colombia. Para mayor información referirse a http://creativecommons.org/licenses/ by-nc-nd/2.5/co/

\section{Tipos de Artículos}

CALJ da la bienvenida a los siguientes tipos de artículos, requeridos por Colciencias:

1) Artículos sobre Reportes de Investigación: Es un documento que detalla los resultados originales de proyectos de investigación. La estructura de este tipo de artículo suele contener los siguientes apartados: título, 
resumen, palabras claves, introducción, marco teórico, metodología, análisis y discusión de los resultados, conclusiones, y referencias. Los autores deberán incluir ayudas gráficas.

2) Artículo de Reflexiones sobre la Praxis: Es un documento que muestra los resultados de una investigación en el aula sobre un tema pedagógico específico desde la perspectiva analítica, interpretativa o crítica, que presenta una innovación con implicaciones pedagógicas. Este tipo de artículo, pueden consistir: consideraciones teóricas, contexto pedagógico, resultados, discusión, contexto, etc. Se Espera que los autores incluyan ayudas gráficas. Debe contener las fuentes originales.

3) Articulo de Revisión de Temas Teóricos: Es un documento derivado de la investigación en la que se publicó los resultados de investigación, o no publicadas, en un campo científico, en la cual se analizan, sistematizan y se integran con el fin de informar a los lectores de las últimas tendencias y desarrollos. Normalmente se hace una revisión cuidadosa de la literatura con al menos 50 referencias. Este tipo de artículo, puede incluir: evolución, factores, perspectivas, contexto, revisión bibliográfica, debate, planteamientos, críticas, etc. Los autores deberán incluir ayudas gráficas.

4) Artículos Cortos: Es un documento breve que presenta preliminarmente o parcialmente resultados originales de un estudio de investigación científica y generalmente, requiere una pronta difusión. Este tipo de artículo, debe incluir: evolución, factores, perspectivas, contexto, implicaciones, etc. Se espera que los autores incluyan ayudas gráficas.

5) Informes de Caso: Este documento presenta los resultados de un estudio sobre una situación particular, con el fin de difundir las experiencias metodológicas y técnicas, utilizadas en un caso concreto. El informe incluye un comentario de la literatura de casos similares. Los autores deberán incluir ayudas gráficas.

6) Revisiones de Tema: Es un documento resultante de una revisión crítica de la literatura relacionada con un tema específico.

7) Reseña del libro: Revisión crítica y analítica, debe proporcionar un contexto para el trabajo en cuestión. No tiene que ser extensa, máximo 2000 palabras, debe responder a las preguntas informativas básicas de un lector. Proporcionar evidencia para respaldar sus opiniones.

\section{Forma y Estilo}

Portada: Incluye el título y el nombre, afiliación institucional, dirección postal completa y dirección de correo electrónico de cada autor en un documento de word separado del artículo. Indicando el coautor a quien debe enviarse la correspondencia. El autor debe incluir el tipo de artículo que está presentando y si el artículo está basado en la investigación original, una tesis de grado, un ensayo o una revisión crítica, debe incluir el título del proyecto, el patrocinador (si es el caso), el código de inscripción del proyecto o el número del contrato respectivo y las fechas, en el caso de artículos originales de investigación, así como la institución asociada al proyecto de investigación.
Resumen: Deben estar incluidos un resumen escrito en inglés y español. Si el autor es incapaz de traducir un resumen en ambos idiomas, por favor indique esto en su presentación. El resumen debe ser entre 150 y 200 palabras. El resumen debe mostrar claramente el problema o la cuestión en estudio, la perspectiva teórica(s) en que el problema se plantea, en la metodología empleada, y, por último, los resultados y conclusiones.

Palabras Clave: Es una lista de cuatro a seis palabras clave para el artículo. Escriba "palabras clave:" en cursiva al final de su resumen. No use negrita. Por favor, incluya las palabras clave en español, así como en inglés.

Ayudas Gráficas: Sugerimos a los autores incluir ayudas gráficas en el contenido de sus artículos. Estas incluyen tablas, esquemas, apéndices, ilustraciones, etc., que ayudan al lector a entender mejor el artículo. Deben incluir las referencias bibliográficas correspondientes. Los autores deben incluir las ilustraciones o ayudas gráficas en un documento de word separado con mínimo 300dpi de resolución. Cuando los autores decidan incluir ayudas gráficas en el texto, éstas se deben enunciar en una línea separada [INSERTAR Ayuda Gráfica nombre AQUí]. Cada ayuda audiovisual debe estar claramente etiquetada con el número y la leyenda. Deben ser numeradas

Figura 1, Figura 2, Tabla 1, Tabla 2, etc. Los gráficos pueden ser modificados o dejados de acuerdo a la decisión del editor y a las necesidades de la publicación. Cualquier cambio será discutido con el autor antes de su publicación.

Longitud: Los artículos normalmente deben estar entre 4.000 y 8.000 palabras (incluyendo el resumen, notas, referencias, tablas, figuras, apéndices y demás elementos). La longitud del manuscrito no debe superar las 10.000 palabras. Las contribuciones que excedan estas longitudes máximas pueden ser consideradas para publicación, en aquellos casos, en que su importancia científica lo justifique.

Requisitos de Software: Todos los textos deberán presentarse en un sistema operativo de Microsoft Word, incluyendo Word 93-2007, Vista o Windows 7.

Idiomas de Publicación: Los documentos son recibidos en inglés o español académico. Las expresiones lingüísticas de otros grupos culturales (es decir, spanglish, jergas, Cockney, afro-americanos, quechuas, etc.) son bienvenidos como muestras y objetos de la investigación.

Bio-datos y anonimato: Todos los artículos pasan por un proceso de revisión por pares y se leen de forma anónima por un miembro del comité de evaluación de CALJ. Por lo tanto, los contribuyentes deberán incluir su nombre completo y afiliación profesional en un documento por separado. En todos los casos se solicita a los autores abstenerse de escribir sus nombres y filiación profesional en el cuerpo del artículo o manuscrito. 
Garantía de Primera Publicación: Los autores deberán firmar una carta reconociendo que el artículo enviado a CALJ no ha sido publicado anteriormente en su forma presente o esencialmente versión similar, ni enviado a otra parte para ser considerado para la impresión o publicación electrónica.

Formulario de Autorización para la Publicación: Los autores de todos los artículos que son aceptados para publicación deberán firmar un formulario de autorización en donde aceptan que el Colombian Applied Linguistics Journal, podrá publicar el artículo sobre una base de datos de acceso abierto. Además, los autores deberán acordar que los artículos publicados en Colombian Applied Linguistics Journal, no pueden ser reproducidos, a menos que soliciten previa autorización para reproducir material publicado. La firma del formulario, es una condición para la publicación y los documentos no serán cedidos a la editorial, a menos que, se firme algún documento de que ha sido recibido.

Compensación: Los autores recibirán dos ejemplares de CALJ, en el cual aparezca su artículo.

Permiso y Consentimiento: Si el artículo contiene extractos de otras obras, especialmente las figuras, tablas, etc., se solicita a los autores contáctarse con los autores y editores de esas obras (los titulares de los derechos de autor), antes de presentar la versión final, para pedir permiso para usar el trabajo. Al enviar el artículo, los autores garantizan que han obtenido permiso del titular de los derechos de autor, para reproducir (en cualquier formato impreso o electrónico), cualquier material no escrito por el autor y que el autor ha reconocido en la fuente. Si en los datos primarios se incluyen participantes en el proyecto de investigación, se requiere obtener un permiso firmado en un formulario de consentimiento de los participantes en el estudio.

Presentaciones: La presentación del artículo debe ser dividida en tres archivos diferentes: la portada, el artículo anonimo para su revisión, y las ayudas gráficas. Los artículos pueden ser enviados por correo electrónico a caljournal. ud@gmail.com o maestria@udistrital.edu.co.

Renuncia: Todos los artículos estarán sujetos a la revisión del comité editorial. El editor se reserva el derecho a realizar modificaciones formales a los artículos en todo el proceso de edición.

Contáctese: Comuníquese con el Journal a caljournal.ud@ gmail.com o llamando al

TEL: (571) 3238400 Ext. 6362 o a través de correo postal a:

CALJ, Editora Maestría en Lingüística Aplicada a la

Enseñanza de Inglés

Universidad Distrital Francisco José de Caldas

Avenida Ciudad de Quito No. 64-81 Oficina 704

Bogotá, Colombia, Sur América

\section{Formato}

Se requiere a todos los autores seguir rigurosamente el Manual de Publicación de American Psychological Association (APA), Sexta Edición, 2010, para el formato apropiado. Para más información utilice los siguientes enlaces:
Guía de Estilo APA:

http://www.apastyle.org/apa-style-help.aspx

Formato y Guía de Estilo APA:

http://owl.english.purdue.edu/owl/resource/560/1/

Ejemplos APA:

http://owl.english.purdue.edu/owl/resource/560/18/

Fuente: Times New Roman, 12 puntos. Doble espacio en todo el documento. Espacios entre las palabras o después de períodos completos y los dos puntos debe ser un espacio.

Márgenes: 1" de margen en cada lado.

Título: Centrado, no use negrita, utilice minúscula, mayúscula solo en la primera letra de cada palabra, excepto en preposiciones, puede usar hasta dos líneas.

Encabezado: Debe aparecer en cada un de la paginas de su documento. Escriba el título de su documento en mayúsculas en el margen superior izquierdo. El número de páginas en forma consecutiva en el margen superior derecho.

Títulos: No más de dos niveles debajo del título. Los títulos no deben ser numerados. El primer nivel del título va en negrita, y minúsculas excepto por la primera letra de cada palabra. Un segundo nivel del título va en cursiva, también, en minúsculas excepto la primera letra de cada palabra. Párrafos: Un espacio de línea se debe dejar entre párrafos. La primera línea de los nuevos párrafos debe tener sangría, con una sangría de $1 / 4 \mathrm{~cm}$. $(1 / 2 ")$. No utilice un retorno al final de una línea en la gestión de texto, salvo en el final de un párrafo.

Saltos de Página: No inserte saltos de página en el texto. No inserte espacios adicionales para evitar las viudas o huérfanos. Los saltos de página serán diferentes en las pruebas de composición tipográfica. No use separación de sílabas y de justificación.

Corrija y revise la ortografía de su trabajo cuando haya terminado. Haga una copia de seguridad de todo su trabajo. En caso de detectar cualquier virus en su máquina, por favor, informar a todos sus contactos en CALJ inmediatamente.

\section{Fuentes de Documentación}

Por favor, siga el formato APA para la documentación adecuada de las fuentes en su papel y de su lista de referencias. Usted puede encontrar los siguientes enlaces útiles:

APA Reference List: http://owl.english.purdue.edu/owl/ resource/560/05/

APA In-Text Citations http://owl.english.purdue.edu/owl/ resource/560/02/

Los siguientes son ejemplos de cómo citar libros, capítulos de libros, artículos, documentos gubernamentales, artículos en procedimiento, artículos de una publicación periódica en línea etc. para la lista de referencias. 


\section{Libro}

Calfee, R. C., \& Valencia, R. R. (1991). APA guide to preparing manuscripts for journal publication.

Washington, DC: American Psychological Association.

\section{Capítulo de un libro}

O'Neil, J. M., \& Egan, J. (1992). Men's and women's gender role journeys: A metaphor for healing, transition, and transformation. In B. R. Wainrib (Ed.), Gender issues across the life cycle (pp. 107-123). New York, NY: Springer.

\section{Artículo de revista}

Harlow, H. F. (1983). Fundamentals for preparing psychology journal articles. Journal of Comparative and Physiological Psychology, 55, 893-896.

\section{Documento gubernamental}

National Institute of Mental Health. (1990). Clinical training in serious mental illness

(DHHS Publication No. ADM 90-1679). Washington, DC:

U.S. Government Printing Office.

\section{Actas de conferencias}

Schnase, J. L., \& Cunnius, E. L. (Eds.). (1995). Proceedings

from CSCL '95: The First International Conference on
Computer Support for Collaborative Learning. Mahwah, NJ: Erlbaum.

\section{Artículo de una Publicación periódica en línea}

Autor, A. A., \& Autor, B. B. (fecha de publicación). Título del artículo. Título de la publicación, volumen número (número de la edición si tiene). Obtenido de: http://www. someaddress.com/full/url/

\section{Pie de Páginas}

Éstas deben guardar un mínimo absoluto y deben estar fijadas al final del texto principal. No use programas automáticos de pie de nota. Las citaciones deben aparecer en el texto.

\section{Referencias en el texto}

Estas deben aparecer en el cuerpo del artículo, sin pie de página, seguido por el apellido del autor, seguido por el año y el número de página cuando sea necesario. Use comillas dobles para citar el material. Cualquier tipo de cita que contenga más de 40 palabras debe ser fijado en un párrafo principal y no necesita comillas.

Para más información refiérase a las normas APA. 


\section{Call for Papers for 2015-2016}

The Colombian Applied Linguistics Journal, CALJ, is published twice a year by the College of Sciences and Education, Master's Program in Applied Linguistics of Universidad Distrital in Bogotá, Colombia for professionals of education interested in research and teaching issues in the field of Applied Linguistics for the Teaching of English. This journal is mainly concerned with disseminating completed and ongoing research relevant to the field of language education. CALJ is indexed in the MLA International Bibliography, Educational Research Abstracts Online (ERA), Latindex, Ulrichs Periodicals Directory, Redalyc, and Colciencias Category A2. The guidelines for writers can be found in the web page of the journal: http//calj.udistrital.edu.co

\section{CALJ is permanently receiving submissions for future issues.}

\section{Deadlines for submissions:}

Volume 17 No. 2: December 19/2014

Volume 18 No. 1: June 20/2015

Volume 18 No. 2: December 19/2015

The submission should be broken into three separate files: the title page, the article which has been blinded for review, and any graphic aides. Articles can be sent via email to caljournal.ud@gmail.com or postal mail to:

CALJ, Editor

Maestría en Lingüística Aplicada a la Enseñanza de Inglés

Universidad Distrital Francisco José de Caldas

Avenida Ciudad de Quito No. 64-81 Oficina 704

Bogotá, Colombia, Sur América 


\section{MEMBERSHIP}

Colombian Applied Linguistics Journal is published twice a year. New and renewal subscriptions and requests for sample copies should be sent to: CALJ Subscriptions, Avda. Cuidad de Quito No. 64-81 Of. 704, Bogotá, Colombia. Tel: 3238400 Ext. 6362. Subscriptions can be made by e-mail to caljournal.ud@gmail.com. Include the personal information and a PDF of your receipt.

Institutional: $\$ 50.000$ annual

\section{Membership Rates}

Personal*: \$30.000 annual.

* Personal rates apply only when copies are sent to a private address and payment is made to

Universidad Distrital. Prices include postage by airmail.

\section{Payment Information}

Payment is required with all orders. Suscriptions can be made to Banco de Occidente, account number 230-81461-8 from Universidad Distrital. Código 8080.

\section{Personal Information}

To renew or subscribe please fill out the form below and include a copy of your receipt.

Name:

Home Address:

City:

Phone Number:

E-mail:

Work Address:

Institution Name:

Institution E-mail:

Address:

City: 


\section{SUSCRIPCIONES}

La revista Colombian Applied Linguisctics se publica semestralmente. Las renovaciones, nuevas suscripciones y solicitudes de copias de muestra deben ser enviadas a: Suscripciones CALJ Avda.

Ciudad de Quito No 64-81 Of. 704, Bogotá, Colombia. Tel: 3238400 Ext.6362. Las suscripciones también pueden hacerse vía e-mail a caljournal.ud@gmail.com. Debe incluir su información personal y un archivo en PDF de su recibo de pago.

Institucional: $\$ 50.000$ suscripción anual

\section{Tarifas de suscripción}

Personal*: \$30.000 suscripción anual

* La tarifa personal aplica solamente cuando los ejemplares son enviados a una dirección privada y el pago es realizado a la Universidad Distrital. Los precios incluyen gastos de envío por correo aéreo.

\section{Información de Pago}

Se requiere el pago con todos los pedidos. El pago de la suscripción debe realizarse en la cuenta del Banco De Occidente No 230-81461-8 a nombre de la Universidad Distrital. Código 8080.

Información Personal

Para renovar su suscripción a la revista debe diligenciar estos datos e incluir una copia de su recibo de pago.

Nombre:

Domicilio:

Ciudad:

Teléfono:

E-mail:

Dirección laboral:

Nombre de la institución:

E-mail Institucional:

Dirección:

Ciudad: 

Colombian Applied Linguistics Journal fue compuesto en caracteres Optima 11/14 puntos,

impreso sobre papel Alternative B C. Noviembre de 2014. Imprenta UD. Bogotá-Colombia 